\section{Bronchoscopic and angiographic findings in tracheobronchial endometriosis}

\author{
Ping-Hung Kuo, Hao-Chien Wang, \\ Yuang-Shuang Liaw, Sow-Hsong Kuo
}

\begin{abstract}
The case is described of a 31 year old woman who presented with complaints of recurrent haemoptysis coinciding with menstruation. Bronchoscopic examination revealed multiple purplish-red submucosal lesions on the right side of the trachea and bilateral bronchial trees which appeared during her menses and regressed in the intermenstrual periods. Brush cytology revealed cell clusters consistent with endometrial origin. Bronchial angiography demonstrated prominent vasculature at the right paratracheal area and bilateral bronchial trees corresponding to the lesions seen on bronchoscopic examination. Her haemoptysis was satisfactorily controlled by danazol therapy and follow up bronchoscopy showed disappearance of the tracheobronchial lesions. To our knowledge this is the first case of thoracic endometriosis with tracheal involvement.

(Thorax 1996;51:1060-1061)
\end{abstract}

Keywords: catamenial haemoptysis, tracheobronchial endometriosis, bronchoscopy, angiography.

Repeated haemoptysis concurrent with menstrual flow ("catamenial haemoptysis") is a rare presentation of thoracic endometriosis, ${ }^{12}$ with fewer than 20 cases having been reported in the English literature. ${ }^{3}$ Since most cases of pulmonary endometriosis involve distal pulmonary parenchyma rather than large airways,${ }^{4}$ the diagnostic yield from bronchoscopic examination is small and no bronchoscopic images have ever been published. We report the bronchoscopic and angiographic findings of tracheobronchial endometriosis in a 31 year old woman who presented with catamenial haemoptysis.

\section{Case report}

A 31 year old unmarried woman was admitted for investigation of recurrent haemoptysis for the previous four months. Her symptoms started on the first day of her menses and spontaneously resolved two or three days later. There was no associated fever or dyspnoea, and she had never had haemoptysis at any other time in her cycle. No prior history of dysmenorrhoea or abnormal vaginal bleeding had been experienced. Menarche occurred at 14 years of age with menses appearing every 30 days. Chest examination was not significant, and the gynaecological consultation revealed no evidence of pelvic or abdominal endometriosis. Sputum smears were negative for acid-fast bacilli and malignant cells. A chest radiograph showed some ill defined infiltrations of the right middle and lower lung fields. The computed tomographic (CT) scan of the chest demonstrated mild thickening of the bronchial wall and peribronchial infiltrates over the right middle lobe. Bronchoscopic examination performed on the second day of her mense disclosed multiple purplish-red submucosal lesions on the right side of the trachea and bilateral bronchial trees with easy touch oozing (fig 1A and B). Increased vascularity adjacent to these lesions was also observed. There were fresh blood clots in the right lower lobe bronchus. Rupture of one tracheal lesion with a brush released several millilitres of blood. Cytological evaluation of the brushing material demonstrated clusters of small round cells consistent with endometrial origin. However, haemorrhage and normal mucosa were found in the biopsy sample obtained. Cultures of bronchial brushings were negative for $\mathrm{Myco}$ bacterium tuberculosis and fungi.

The patient underwent bronchial angiography which demonstrated prominent vasculature and vascular stains in the right paratracheal area (fig 2) and bilateral bronchial trees corresponding to the lesions seen on bronchoscopy. Follow up bronchoscopy in the middle of her cycle showed regression of previous tracheobronchial lesions. The next episode of haemoptysis concurred expectedly with her menstruation two weeks later, whereupon bronchoscopic examination disclosed recurrence of purplish tracheobronchial lesions as well as multiple foci of hypervascularity. Brush cytology again revealed distinctive features of endometrial cells.

Danazol was administered at an initial dose of $400 \mathrm{mg}$ per day and treatment was continued for eight months while she remained amenorrhoeic. A minor recurrence of haemoptysis occurred in the third month of treatment,
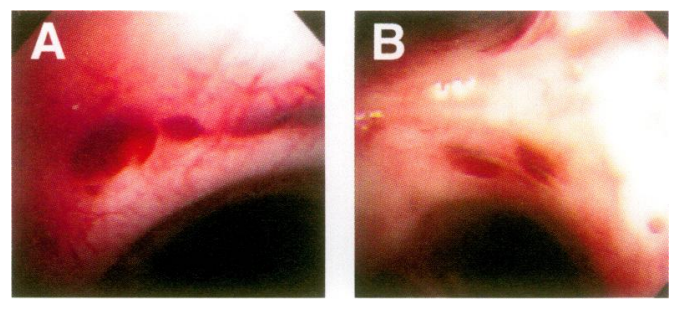

Figure 1 Bronchoscopic examination showing purplishred submucosal lesions with easy touch oozing on $(A)$ the lower trachea and $(B)$ the left secondary carina. These lesions appeared during the menstruation period but regressed in the middle of the cycle. 
Figure 2 Right bronchial angiogram revealing hypervascularity over the right paratracheal area.

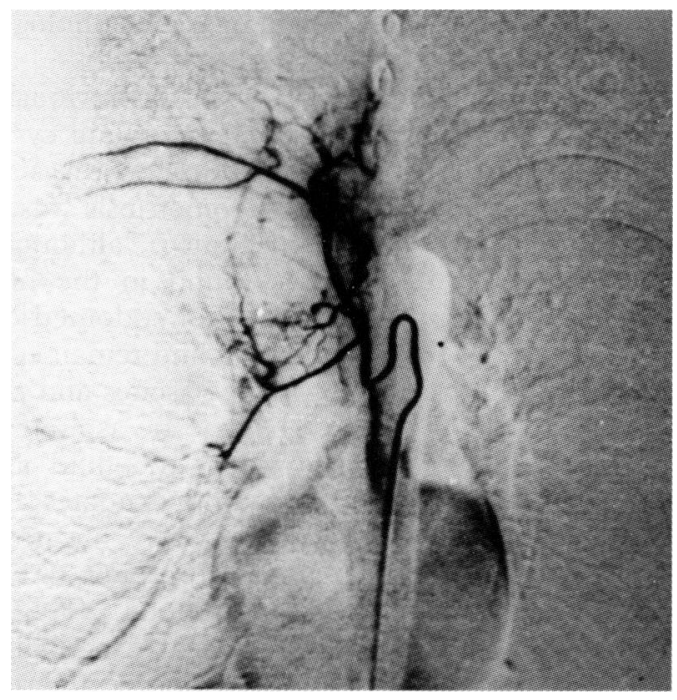

whereupon bronchoscopic examination revealed mild oozing from the right lower lobe but the previous lesions on the trachea and left bronchial tree had disappeared. Some depressed scars on the endobronchial surface of the left main bronchus and left upper lobe were also observed. Unfortunately, her haemoptysis recurred three weeks after danazol was discontinued, but bronchoscopic examination did not reveal significant findings. Danazol treatment was reinstituted with the dosage increased to $600 \mathrm{mg}$ per day, after which no further haemoptysis was reported and she remains asymptomatic to the present time.

\section{Discussion}

Haemoptysis coincident with menstrual periods helps to differentiate catamenial haemoptysis from haemoptysis of other causes. The diagnosis of catamenial haemoptysis is often clinical. ${ }^{4}$ Chest radiographs are often normal, but may reveal solitary or multiple pulmonary nodules with cyclical changes in size. CT findings of pulmonary endometriosis may include ill-defined or well-defined opacities, nodular lesions, thin-walled cavities, or bullous formations. ${ }^{56}$ Elliot et al suggested that CT scanning is the method of choice in confirming the diagnosis provided that it is performed during the period of haemoptysis. ${ }^{4}$ The diagnostic yield from bronchoscopy is small since most pulmonary endometriosis involves distal pulmonary parenchyma rather than the mucosa of the large bronchi and the washings and biopsy specimens often yield inconclusive results. ${ }^{7}$ Although definite histological evidence of endometriosis was not obtained in this case, the cytological features as well as the cyclical changes in the bronchoscopic findings were sufficient to warrant the diagnosis. In addition, the importance of proper timing of bron- choscopy with the occurrence of haemoptysis cannot be overemphasised because endometrial cysts may rupture and heal. ${ }^{2}$ To our knowledge, this is the first case of thoracic endometriosis with tracheal involvement to be reported in the literature.

Another striking feature of this case was the angiographic findings which have not been previously demonstrated in thoracic endometriosis. In 1990 Katoh et al suggested that angiography was of little value in the evaluation of pulmonary endometriosis. ${ }^{5}$ In our patient bronchial angiography revealed prominent vasculature and vascular stains which corresponded to the endometrial lesions seen on bronchoscopy. Although we did not perform the follow up angiography during her intermenstrual period, these findings suggested the association of endometriosis with the cyclical occurrence of endobronchial hypervascularity. Whether this vascular change was directly related to endometriosis remains unclear. Bronchial endometriosis was suggested as a cause of bronchiectasis which developed in a patient after cyclical haemoptysis lasting 30 years. $^{8}$ The proposed mechanisms linking endometriosis with bronchiectasis include recurrent bleeding into the interstitium with subsequent traction and changes in the airways and secondary infection in the area of bleeding. ${ }^{8}$ Multiple depressed scars on the endobronchial surface, as observed in our patient after three months of treatment with danazol, have also been described in a previous report. ${ }^{2}$ The long term pulmonary consequences of repeated haemoptysis in endometriosis remain to be determined.

Current treatment regimens of pulmonary endometriosis include danazol and gonadotropin releasing hormone (GnRH) analogues. ${ }^{910}$ The results obtained with danazol suggested an improvement in the course of the disease, although the incidence of recurrence was high. ${ }^{9}$ Bronchoscopic studies also confirmed the efficacy of danazol in this rare cause of haemoptysis.

1 Rodman $\mathrm{MH}$, Jones CW. Catamenial hemoptysis due to bronchial endometriosis. N Engl f Med 1962;266:805-8.

2 Bateman ED, Morrison SC. Catamenial haemoptysis from endobronchial endometriosis: a case report and review of previous reported cases. Respir Med 1990;84:157-61.

3 Wood DJ, Krishnan K, Stocks P, Morgan E, Ward MJ. Cood DJ, Krishnan K, Stocks P, Morgan E, Ward MJ.
Catamenial haemoptysis: a rare cause. Thorax 1993;48: 1048-9.

4 Elliot DL, Barker AF, Dixon LM. Catamenial hemoptysis. New methods of diagnosis and therapy. Chest 1985;87: 687-8.

5 Katoh O, Yamada H, Aoki Y, Matsumoto S, Kudo S. Utility of angiograms in patients with catamenial hemoptysis. Chest 1990;98:1296-7.

6 Volkart JR. CT findings in pulmonary endometriosis. $f$ Comput Assist Tomogr 1995;19:156-9.

7 Guidry GG, George RB. Diagnostic studies in catamenia hemoptysis. Chest 1990;98:260-1.

8 Butler H, Lake KB, Van Dyke JJ. Bronchial endometriosis and bronchiectasis. Arch Intern Med 1978;138:991-2.

9 Johnson WM, Tyndal CM. Pulmonary endometriosis: treatment with danazol. Obstet Gynecol 1987;69:506-7.

10 Espaulella J, Armengol J, Bella F, Lain JM, Calaf J. Pulmonary endometriosis: conservative treatment with GnRH agonists. Obstet Gynecol 1991;78:535-7. 